\title{
Spacer acquisition mechanism in type II-A CRISPR system.
}

\author{
Yibei Xiao ${ }^{1}$, Jagat Budhathoki ${ }^{1}$, Sherwin $\mathrm{Ng}^{1, \#}, \mathrm{Ki}^{\mathrm{H}}$ yun Nam${ }^{2, \#}$, Ailong $\mathrm{Ke}^{1, *}$ \\ ${ }^{1}$ Department of Molecular Biology and Genetics, Cornell University, 253 Biotechnology \\ Building, Ithaca, NY 14853, USA. \\ ${ }^{2}$ Pohang Accelerator Laboratory, Pohang University of Science and Technology, \\ Pohang, South Korea.
}

Molecular memory is created when a short foreign DNA-derived prespacer is integrated into the CRISPR array as a new spacer. Whereas the RNA-guided CRISPR interference mechanism varies widely among CRISPR-Cas systems, the spacer integration mechanism is essentially identical. The conserved Cas 1 and Cas 2 proteins form an integrase complex consisting of two distal Cas1 dimers bridged by a Cas 2 dimer. The prespacer is bound by Cas1-Cas2 as a dual-forked DNA, and the terminal 3'-OH of each 3 ' overhang serves as an attacking nucleophile during integration. The prespacer is preferentially integrated into the leader-proximal region of the CRISPR array, guided by the leader sequence and a pair of inverted repeats inside the CRISPR repeat. Spacer integration in the well-studied Escherichia coli type I-E CRISPR system also relies on the bacterial integration host factor. In type II-A CRISPR, however, Cas1-Cas2 alone integrates spacers efficiently in vitro; other Cas proteins (such as Cas9 and Csn2) have accessory roles in the biogenesis phase of prespacers. I will present our structural and biochemical efforts in revealing the spacer acquisition mechanism in the Enterococcus faecalis type II-A CRISPR system. 